\title{
Absorption suppression in photonic crystals
}

\author{
A. Figotin and I. Vitebskiy
}

\begin{abstract}
We study electromagnetic properties of periodic composite structures, such as photonic crystals, involving lossy components. We show that in many cases a properly designed periodic structure can dramatically suppress the losses associated with the absorptive component, while preserving or even enhancing its useful functionality. As an example, we consider magnetic photonic crystals, in which the lossy magnetic component provides nonreciprocal Faraday rotation. We show that the electromagnetic losses in the composite structure can be reduced by up to two orders of magnitude, compared to those of the uniform magnetic sample made of the same lossy magnetic material. Importantly, the dramatic absorption reduction is not a resonance effect and occurs over a broad frequency range covering a significant portion of the respective photonic frequency band.
\end{abstract}




\section{Introduction}

Magnetic materials play a crucial role in microwave technology and optics. They are absolutely essential in numerous nonreciprocal devices such as isolators, circulators, phase shifters, etc. They can also provide tunability, miniaturization, better impedance matching, and other important features. A major obstacle for broader applications of magnetic materials is tied up with the issue of absorption. Many magnetic materials with otherwise perfect physical characteristics have been rejected because of strong losses at frequency range of interest. In this paper we explore the idea of composite magnetic structures having desired physical properties associated with magnetism but, at the same time, significantly suppressing the effects of absorption. In other words, we want to take advantage of the useful characteristics of a particular magnetic material, while drastically reducing its contribution to the energy dissipation.

Magnetic photonic crystals are spatially periodic composite structures with one of the components being a magnetic material, such as a ferromagnet or a ferrite. Extensive information on the subject and numerous references can be found in a recent review article [1. Similarly to other photonic crystals, magnetic photonic crystals display strong spatial dispersion, resulting in appearance of electromagnetic band-gap structure. But in addition, magnetic photonic crystals can provide tunability and better impedance matching than regular non-magnetic photonic crystals [2].

In comparison to uniform magnetic materials, magnetic photonic crystals can display much stronger nonreciprocal properties, such as magnetic Faraday rotation 1. 2, 3, 4, 5, 6, 7, 8, 9. Strong nonreciprocal effects are essential in isolators, circulators, and other microwave and optical devices. The possibility of appreciable enhancement of Faraday rotation is particularly important at infrared and optical frequencies, where the non-reciprocal effects in uniform magnetic materials are very weak. In addition, the use of periodic structures instead of uniform magnetic materials can dramatically reduce the size of non-reciprocal and other microwave and optical devices.

The subject of our investigation is another important aspect of electrodynamics of periodic composite structures. Namely, we show that properly design periodic array can dramatically reduce the losses associated with individual constitutive components. In the particular case of magnetic photonic crystals, the broadband suppression of losses can be achieved in a combination with the enhancement of nonreciprocal properties, such as Faraday rotation, linear magnetoelectric response, etc. The possibility of the reduction of losses is related to the fact that in most cases the absorption and the useful functionality of the particular magnetic material are related to different components of its permittivity and/or permeability tensors $\hat{\varepsilon}$ and $\hat{\mu}$. Specifically, the absorption is determined by the anti-Hermitian parts $\hat{\varepsilon}^{\prime \prime}$ and $\hat{\mu}^{\prime \prime}$ the permittivity and permeability tensors

$$
\hat{\varepsilon}^{\prime \prime}=-\frac{i}{2}\left(\hat{\varepsilon}-\hat{\varepsilon}^{\dagger}\right), \hat{\mu}^{\prime \prime}=-\frac{i}{2}\left(\hat{\mu}-\hat{\mu}^{\dagger}\right),
$$

where $\dagger$ denotes Hermitian conjugate. By contrast, the nonreciprocal circular birefringence responsible for the Faraday rotation is usually determined by the Hermitian skew-symmetric part of the respective tensors

$$
\hat{\varepsilon}_{a}=\frac{1}{2}\left(\hat{\varepsilon}-\hat{\varepsilon}^{T}\right), \hat{\mu}_{a}=\frac{1}{2}\left(\hat{\mu}-\hat{\mu}^{T}\right),
$$


where the subscript $T$ denotes matrix transposition. The relations (1) and (2) suggest that the rate of energy absorption by the lossy material can be functionally different from its useful functionality (nonreciprocal circular birefringence in our case). Such a difference allows us to adjust the physical and geometric characteristics of the periodic structure so that the electromagnetic field distribution inside the photonic crystal suppresses the energy dissipation by the lossy magnetic component, while preserving or even enhancing its useful functionality. Moreover, in some cases, a sufficiently strong absorption can affect the electromagnetic field distribution in such a way that it suppresses its own contribution to the total rate of absorption of the composite material. In the latter situation, the stronger absorption coefficient of the lossy component is, the less it contributes to the total rate of absorption of the composite structure.

The way to address the problem of absorption suppression in a periodic composite structure essentially depends on the following three factors.

1. The useful functionality of the lossy material. In our example, it will be the nonreciprocal circular birefringence producing the Faraday rotation.

(i) The dominant physical mechanism of absorption. For instance, energy dissipation caused by electric conductivity requires a different approach, compared to the situation where the losses are associated with the dynamics of magnetic domains, or some other physical mechanisms. In each individual case, the structure of the anti-Hermitian part (1) of the permittivity and/or permeability tensors can be different, and so can be the optimal configuration of the composite material.

(ii) The frequency range of interest. The same periodic array can significantly reduce losses at some frequencies, while enhancing losses at different frequencies. In other words, the same periodic structure can be either effective or counterproductive, depending on the frequency range and the dominant physical mechanism of electromagnetic energy dissipation.

The possibility of a significant absorption reduction is not limited to magnetic composites. Similar approach can be applied to other heterogeneous structures with lossy components. A key requirement is that the useful functionality of the lossy material should be functionally different from its contribution to the energy dissipation. In such a case, the periodic structure can be engineered so that at frequency range of interest, the electromagnetic field distribution inside the composite medium suppresses the losses, while preserving the useful functionality of the lossy component. This can always be achieved if the components of the tensors $\hat{\varepsilon}$ and $\hat{\mu}$ related to the useful functionality of the lossy material are different from those dominant in the anti-Hermitian tensors $\hat{\varepsilon}^{\prime \prime}$ and $\hat{\mu}^{\prime \prime}$. In any event, in order to suppress the energy dissipation caused by lossy component of the periodic structure we have to take into account the physical nature of absorption, the useful functionality of the lossy material, and the frequency range of interest.

In the next section we produce a specific numerical example of a periodic structure that includes a lossy magnetic material. The amount of Faraday rotation produced by this periodic structure is comparable to that of a uniform slab made of the same lossy magnetic material. But the absorption rate of the composite structure is 20 to 100 times lower than that of the uniform magnetic slab. Importantly, such a dramatic absorption reduction is achieved in a broad frequency range covering almost an entire photonic frequency band. Another practical advantage is that in some cases the size 
of the composite structure can be much smaller compared to that of the uniform magnetic sample with similar characteristics. Note, though, that a significant size reduction usually comes at the expense of the bandwidth.

\section{Absorption suppression in a periodic layered structure}

Consider a monochromatic plane wave normally incident on a uniform magnetic slab, as shown in Fig. 1 Suppose that the useful functionality of the slab is the nonreciprocal Faraday rotation. This is the case with almost all nonreciprocal microwave and optical devices, such as the isolators, circulators, etc. The statement of the problem is as follows. On the one hand, we have a plane-parallel uniform magnetic slab characterized by certain Faraday rotation and absorption. This uniform slab is shown in Fig. 1. On the other hand, we have a stack of layers made of the same lossy magnetic material alternating with some other layers. Such a stack is shown in Fig. 2 We expect the properly designed periodic stack in Fig. 2 to be superior to the uniform magnetic slab in Fig. 1. The superiority can be defined by the following set of requirements.

1.1 The stack produces similar or larger Faraday rotation, as compared to that of the uniform magnetic slab. This means that the stack and the uniform slab have comparable useful functionality.

1.2 The stack has much lower absorption than the uniform magnetic slab. This requirement reflects our prime objective.

1.3 The stack dimensions do not exceed those of the uniform magnetic slab.

1.4 The stack displays all the above properties within a reasonably broad frequency range.

The above set of requirements corresponds to a broadband absorption suppression. Alternatively, we can impose a slightly different set of requirements.

2.1 The stack produces similar or larger Faraday rotation in comparison to the uniform magnetic slab.

2.2 The stack has much lower absorption than the uniform magnetic slab.

2.3 The stack has much smaller dimensions than the uniform magnetic slab.

Notice, that the requirement 2.3 of much smaller dimensions comes at the expense of the bandwidth (the requirement 1.4).

In this section we provide numerical examples proving the effectiveness of the photonic crystal approach to absorption suppression. In all cases we assume that the permittivity and permeability tensors of the magnetic material have the following standard form

$$
\hat{\varepsilon}_{F}=\left[\begin{array}{ccc}
\varepsilon_{1}+i \gamma_{e} & i \alpha & 0 \\
-i \alpha & \varepsilon_{1}+i \gamma_{e} & 0 \\
0 & 0 & \varepsilon_{3}
\end{array}\right], \quad \hat{\mu}_{F}=\left[\begin{array}{ccc}
\mu_{1}+i \gamma_{m} & i \beta & 0 \\
-i \beta & \mu_{1}+i \gamma_{m} & 0 \\
0 & 0 & \mu_{3}
\end{array}\right] \text {, }
$$

corresponding to either uniaxial or isotropic magnetic material placed in a bias magnetic field parallel to the $z$ axis [10, 11. The quantities $\alpha$ and $\beta$ in (3) are responsible for nonreciprocal circular birefringence [10. If the direction of magnetization is changed for the opposite, the parameters $\alpha$ and $\beta$ will also change 
sign and so will the sense of Faraday rotation. Usually, at microwave frequencies, $|\beta| \gg|\alpha|$, while at optical frequencies, $|\beta| \ll|\alpha|$. The positive parameters $\gamma_{e}$ and $\gamma_{m}$ in (3) are responsible for absorption. We can assume, for example, that the dominant physical mechanism of absorption is the electric conductivity $\sigma$, which is often the case at microwave frequencies. In a transverse electromagnetic wave, the effect of electric conductivity reduces to the following anti-Hermitian contribution to the electric permittivity tensor $\hat{\varepsilon}_{F}[10$

$$
i \gamma_{e}=4 \pi \sigma i / \omega
$$

Different absorption mechanisms could also result in the dominance of the parameter $\gamma_{m}$ in (3), rather than $\gamma_{e}$. In our numerical examples we only consider the case of $\gamma_{e} \gg \gamma_{m}$.

The uniform magnetic slab in Fig. 1 has the thickness $D_{U}$ and the permittivity and permeability tensors (3). In Fig. 2 we show a setting similar to that of Fig. 1 , but the uniform magnetic slab is now replaced with the periodic layered structure composed of alternating magnetic and dielectric layers. The magnetic $F$ layers are made of the same lossy magnetic material as the uniform slab in Fig. 1. The respective permittivity and permeability tensors are given in (3). The non-magnetic $A$ layers are made of lossless isotropic dielectric material with the permittivity and permeability tensors

$$
\hat{\varepsilon}_{A}=\left[\begin{array}{ccc}
\varepsilon_{0} & 0 & 0 \\
0 & \varepsilon_{0} & 0 \\
0 & 0 & \varepsilon_{0}
\end{array}\right], \quad \hat{\mu}_{A}=\left[\begin{array}{lll}
1 & 0 & 0 \\
0 & 1 & 0 \\
0 & 0 & 1
\end{array}\right] .
$$

The role of the $A$ layers is to create the proper electromagnetic field distribution, which would suppress the energy absorption in the lossy $F$ layers, while preserving or even enhancing the non-reciprocal effects associated with magnetism. The total number of the double layers $L$ in the periodic stack is $N$. The thicknesses $d_{A}$ and $d_{F}$ of the $A$ and $F$ layers satisfy the relation $d_{A}+d_{F}=L$. The total thickness $D_{S}$ of the periodic stack is the product $N L$. We assume that in all cases the thickness $D_{S}$ of the periodic stack in Fig. 2 does not exceed the thickness $D_{U}$ of the uniform magnetic slab in Fig. 1.

The key to the possibility of absorption suppression in the composite structure is that the nonreciprocal effects on the one hand, and the energy dissipation on the other hand, are determined by different components of the material tensors (3). This allows to design the periodic array so that at the frequency range of interest, the electromagnetic field distribution inside the composite structure will favor the nonreciprocal components of the permittivity/permeability tensors responsible for the desired Faraday rotation, while not engaging with the anti-Hermitian components responsible for absorption.

Computations of electromagnetic field distribution inside the stratified and uniform media, as well as the amplitude and polarization of the transmitted and reflected waves are based on the time-harmonic Maxwell equations

$$
\nabla \times \vec{E}(z)=i \frac{\omega}{c} \hat{\mu}(z) \vec{H}(z), \nabla \times \vec{H}(z)=-i \frac{\omega}{c} \hat{\varepsilon}(z) \vec{E}(z),
$$

with $z$ - dependent permittivity and permeability tensors defined in (3) and (5). The electric conductivity $\sigma$ is included in the definition (3) of the respective permittivity tensor $\hat{\varepsilon}_{F}$, as specified in (44). In all cases, the incident wave $\Psi_{I}$ propagates along 
the $z$ direction normal to the layers and has linear polarization with $\vec{E}_{I} \| x$. Due to the nonreciprocal circular birefringence of the magnetic material, the transmitted and reflected waves $\Psi_{P}$ and $\Psi_{R}$ will be elliptically polarized with the ellipse axes being at an angle with the $x$ direction. The magnitude of nonreciprocal effects can be characterized by the $y$ component $\left(\vec{E}_{P}\right)_{y}$ of the transmitted wave $\Psi_{P}$. Indeed, in the absence of magnetism, the parameters $\alpha$ and $\beta$ in (3) vanish and the transmitted wave will be linearly polarized with $\vec{E}_{P} \| x$. This is true both in the case of the uniform magnetic slab in Fig. 1 and in the case of the layered structure in Fig. 2,

The transmission and reflection coefficients of the slab (either uniform, or layered) are defined as follows

$$
t=\frac{S_{P}}{S_{I}}, r=-\frac{S_{R}}{S_{I}},
$$

where $S_{I}, S_{P}$, and $S_{R}$ are the Poynting vectors of the incident, transmitted, and reflected waves, respectively. The slab absorption is

$$
a=1-t-r .
$$

If the incident wave polarization is linear, the coefficients $t, r$, and $a$ are independent of the orientation of vector $\vec{E}_{I}$ in the $x-y$ plane. Assuming that the incident wave $\Psi_{I}$ is linearly polarized with $\vec{E}_{I} \| x$, the magnitude of the nonreciprocal effect can be characterized by the ratio

$$
\rho=\frac{\left(E_{P}\right)_{y}}{\left(E_{I}\right)_{x}}, \quad \text { where }|\rho|<1 .
$$

Generally, the transmitted wave polarization in Figs. 1] and 2 is elliptical, rather than linear. Therefore, the quantity $\rho$ in (9) is not literally the sine of the Faraday rotation angle. The nonreciprocal effects in the scattering problem of Figs. 1]and 2] are more complicated than a simple Faraday rotation. Let us elaborate on this point. The electromagnetic eigenmodes of the uniform magnetic slab in Fig. 11and the eigenmodes of the layered structure in Fig. 2 are all circularly polarized. This implies that if the polarization of the incident wave is circular, the transmitted and reflected waves will also be circularly polarized, both in the case of a uniform slab and in the case of a layered stack, with or without absorption. On the other hand, due to the nonreciprocal (magnetic) effects, the transmission/reflection coefficients for the right-hand circular polarization are different from those for the left-hand circular polarization. This is true regardless of the presence or absence of absorption. Consider now a linearly polarized incident wave. It can be viewed as a superposition of two circularly polarized waves with equal amplitudes. Sine the transmission/reflection coefficients for the righthand and left-hand circular polarizations are different, the transmitted and reflected waves will be elliptically polarized. Such an ellipticity develops both in the case of a uniform slab and in the case of a layered stack, with or without absorption. Note, though, that at optical frequencies, the dominant contribution to the ellipticity of transmitted wave is usually associated with absorption, which is largely responsible for circular dichroism. Without absorption, the ellipticity of the wave transmitted through magnetic the slab in Fig. 11 would be negligible. This is not the case, though, at microwave frequencies, where the ellipticity of transmitted and reflected waves can be significant even in the absence of absorption.

To avoid confusion, note that a linear polarized wave propagating in a uniform, lossless, unbounded, magnetic medium (3) will not develop any ellipticity. Instead, it 
will display a pure Faraday rotation. But the slab boundaries in Fig. 1 and the layer interfaces in Fig. 2 will produce some ellipticity even in the case of lossless magnetic material. The absorption provides an additional contribution to the ellipticity of transmitted and reflected waves. The latter contribution is referred to as circular dichroism. The following numerical examples illustrate some of the above statements.

For simplicity, in further consideration we will often refer to the quantity $\rho$ in (9) as the amount of (nonreciprocal) Faraday rotation, although, due to the ellipticity, it is not exactly the sine of the Faraday rotation angle.

In all plots, the frequency $\omega$ and the Bloch wave number $k$ are expressed in dimensionless units of $c L^{-1}$ and $L^{-1}$, respectively. In our computations we use a transfer matrix approach identical to that described in Ref. [6, 7].

\subsection{Broadband absorption suppression}

We start with the following set of numerical values

$$
\begin{gathered}
d_{A}=0.8 L, d_{F}=0.2 L, N=8, D_{S}=8 L, D_{U}=10 L \\
\varepsilon_{1}=2.89, \mu_{1}=32.49, \varepsilon_{0}=26.01 \\
\alpha=0, \beta=4.0, \quad \gamma_{e}=0.1, \gamma_{m}=0 .
\end{gathered}
$$

The value $\gamma_{e}=0.1$ corresponds to a relatively strong absorption which can be associated with the electric conductivity of the magnetic material. According to (10), the thickness $D_{S}$ of the periodic stack is somewhat smaller than the thickness $D_{U}$ of the uniform magnetic slab. In addition, that the combined thickness $N d_{F}$ of all magnetic layers in the stack is 6 times smaller than the thickness $D_{U}$ of the uniform magnetic slab. The latter implies that not only the periodic stack in Fig. 2 is thinner than the uniform magnetic slab in Fig. 1, but the actual amount of magnetic material used in the composite structure is just a one sixth of that used in the uniform slab.

In Fig. 3 we present a fragment of the $k-\omega$ diagram of the periodic array of layers described in (10); only the lowest photonic frequency band is shown. The split of the two spectral branches is due to the strong circular birefringence $\beta=4$ in (10). In Fig. 4 we show the transmission dispersion of the periodic magnetic stack composed of 8 unit cells $L$. The strong polarization dependence of the stack transmission is also caused by the large value of the nonreciprocal parameter $\beta$ in (10).

Let us now compare the performance of the uniform magnetic slab in Fig. 1 and the periodic stack in Fig. 2, Fig. 5(a) shows that the magnitude $|\rho|$ of the Faraday rotation produced by the periodic stack is comparable to that of the uniform slab. In fact, the periodic stack produces even stronger nonreciprocal effect. At the same time, Fig. 5(b) shows that within the same broad frequency range, the energy dissipation in the layered structure is 20 to 50 times lower, compared to the uniform slab. Hence, the composite structure does indeed dramatically reduce the losses while enhancing the useful functionality of the magnetic material and reducing the overall dimensions. In other words, by all accounts, the layered structure is by far superior to the uniform magnetic slab.

The numerical parameters (10) used in our computations are hypothetical, although realistic. We did not try to optimize the configuration of the periodic layered structure, or to see if the periodic arrays with two or three dimensional periodicity can produce even better results. Our goal here is to prove that even a simple periodic array can dramatically improve the situation with losses. A key is the proper configuration of the composite structure, which, in turn, essentially depends on the physical nature 
of absorption and the frequency range of interest. Also, we would like to emphasize that the dramatic absorption reduction seen in Fig. 5 is not a resonance effect. This is why the suppression of losses is achieved within a relatively broad frequency range and the results are not particularly sensitive to the number of layers in the periodic structure. By contrast, the possibility of a significant size reduction discussed in the next subsection is associated with the transmission band edge resonance, which is usually characterized by a relatively narrow bandwidth and strong dependence on the number of layers in the periodic stack.

\subsection{Resonant absorption suppression}

Under what circumstances can we not only suppress the absorption but also have the size of the periodic composite structure much smaller than that of the uniform (magnetic) slab with similar performance? When considering this question we should keep in mind that within the framework of the photonic crystal approach the characteristic length $L$ of the periodic array is always comparable to that of the electromagnetic wavelength in the medium. Therefore, for a given frequency range and for a given set of the constitutive materials, we cannot significantly change the structural period $L$. Nor can we substantially reduce the number $N$ of unit cells without loosing all the effects of coherent interference. For instance, in our numerical example we have chosen $N=8$, and there is very little room for further reduction in size. All we can achieve by adjusting the configuration of the periodic array comprising as few as several periods is to suppress the losses and/or to enhance the useful functionality, such as Faraday rotation. The real question is: what is the thickness $D_{U}$ of the uniform slab with the useful functionality comparable to that of the optimized layered structure? Indeed, if such a uniform slab turns out to be much thicker than the layered structure, then we can claim that not only the periodic array dramatically reduces the losses, but it also has much smaller dimensions. The latter is only possible if the thickness $D_{U}$ of the uniform slab with desired functionality is much greater than the electromagnetic wavelength in the medium. Otherwise, all we can achieve by introducing periodic inhomogeniety is a significant reduction of losses which, by the way, has been our primary objective.

Let us turn back to the periodic stack in Fig. 2 with the permittivity and permeability tensors of the $F$ and $A$ layers given in (3) and (5). In the numerical example (10), we assume a relatively strong circular birefringence $\beta=4.0$ of the magnetic material. The resulting Faraday rotation in a uniform magnetic slab is of the order of unity even if the magnetic slab thickness is equal to just several electromagnetic wavelengths. Such a small thickness is comparable to the thickness of a photonic structure (a periodic stack) comprising as few as several unit cells $L$. Therefore, in the case (10) of a strong circular birefringence of the magnetic material, the periodic stack cannot have significantly smaller size in comparison to the uniform magnetic slab producing similar nonreciprocal Faraday rotation. The composite structure in this case is only useful for absorption reduction, as we have demonstrated in the previous subsection.

A strong circular birefringence usually occurs in the vicinity of a ferromagnetic resonance at microwave frequency range. If the frequency $\omega$ is much higher or much lower than that, the gyrotropic parameter $\beta$ is much smaller. For instance, at infrared and optical frequencies, in order to produce Faraday rotation $\rho$ of the order of unity, the thickness of the uniform magnetic slab should exceed the electromagnetic wavelength 
by at least two or three orders of magnitude. By contrast, the periodic magnetic stack incorporating the same magnetic material can be much thinner (of the order of several electromagnetic wavelengths), while producing Faraday rotation $\rho$ of the order of unity and dramatically reducing the absorption. In other words, the composite structure with the proper configuration can suppress losses and be much smaller than the uniform sample, but only if the circular birefringence $\beta$ of the magnetic material is relatively weak, specifically, if

$$
\beta \ll \mu_{1} .
$$

To illustrate this point, let us consider the following set of numerical values describing the uniform magnetic slab in Fig. 1 and the periodic magnetic stack in Fig. 2

$$
\begin{gathered}
d_{A}=d_{F}=0.5 L, N=8, D_{S}=8 L, D_{U}=40 L, \\
\varepsilon_{1}=2.89, \mu_{1}=32.49, \varepsilon_{0}=26.01 \\
\alpha=0, \beta=0.1, \quad \gamma_{e}=0.01, \gamma_{m}=0 .
\end{gathered}
$$

Again, the $F$ layers of the periodic array are made of the same lossy magnetic material as the uniform magnetic slab. The Bloch dispersion relation of the respective periodic structure is shown in Fig. 6. There are two major differences between the numerical values in (10) and (11). In the latter case, the value $\beta$ of specific Faraday rotation of the magnetic material is 40 times smaller. To partially offset the weaker circular birefringence and still have the circular birefringence $\rho$ of the order of unity, the thickness $D_{U}$ of the uniform magnetic slab is set to be much larger $(40 L)$. By contrast, properly designed periodic array can have just several unit cells $L$ and sill produce the desired nonreciprocal effect. All the above can be achieved if the frequency is close enough to one of the transmission band edge resonances, also known as slow wave Fabry-Perot resonances. Two of such resonances are shown in Fig. 7. As we shall see below, in the case (11) we can suppress the losses and reduce the size at the same time, but all this comes at the expense of the bandwidth. Specifically, the bandwidth of the desired effect of strong Faraday rotation in a combination with a significant absorption suppression is now limited by the width of the respective transmission band edge resonance. By contrast, in the case (10) described in Fig. 5 , the dramatic reduction of absorption occurs within a broad frequency range spanning a significant portion of the lowest photonic band.

A graphic illustration of the effect of resonance absorption suppression is presented in Fig. 8. A sharp peak in the frequency dependence of the Faraday rotation $\rho$ in Fig. 8(a) coincides with the transmission band edge resonance seen in Fig. 7). Observe that the dramatic enhancement of the Faraday rotation in Fig. 8(a) only occurs in the vicinity of the transmission band edge resonance and is characterized by a relatively narrow bandwidth. Away from the resonance, the stack performance is now mixed. This situation is typical for different resonance and slow-wave phenomena, such as the frozen mode regime [7, 12, 13, 14. By contrast, in our previous example (10), the absorption suppression occurred within a broad frequency range and it was not related to any resonance.

\section{Conclusion}

We have shown that periodic composite structures, such as photonic crystals, can be used to dramatically suppress the energy dissipation associated with the presence of lossy component. In most cases, the useful functionality of the lossy component can 
be preserved or even enhanced by the presence of spatially periodic inhomogeniety. In our first example described in Fig. 15, the absorption suppression by the periodic structure is not a resonance effect. As a consequence, it occurs in a relatively broad frequency range and it is not particularly sensitive to the size and shape of the photonic crystal. The optimal parameters of the periodic structure depend on: (i) the useful functionality of the lossy material, such as Faraday rotation, nonlinearity, etc., (ii) the physical nature of absorption, and (iii) the frequency range of interest. In addition to a significant reduction of losses, the properly designed periodic array can also have much smaller dimensions, compared to the uniform sample with similar functionality. But the size reduction is possible only in special cases and it comes at the expense of the bandwidth, as illustrated in Fig. 8

Acknowledgments: Effort of A. Figotin and I. Vitebskiy is sponsored by the Air Force Office of Scientific Research, Air Force Materials Command, USAF, under grant number FA9550-04-1-0359. 
[1] M. Inoue, et all. Magnetophotonic crystals (Topical Review). J. Phys. D: Appl. Phys. 39, R151R161 (2006).

[2] I. Lyubchanskii1, N. Dadoenkova1, M. Lyubchanskii1, E. Shapovalov, and T. Rasing. Magnetic photonic crystals. J. Phys. D: Appl. Phys. 36, R277-R287 (2003)

[3] M. Inoue, K. Arai, T. Fuji, and M. Abe. One-dimensional magnetophotonic crystals. J. Appl. Phys. 85, 5768 (1999).

[4] M. Levy and A. A. Jalali. Band structure and Bloch states in birefringent onedimensional magnetophotonic crystals: an analytical approach. J. Opt. Soc. Am. B, 24, 1603-1609 (2007).

[5] M. Levy and R. Li. Polarization rotation enhancement and scattering mechanisms in waveguide magnetophotonic crystals. Appl. Phys. Lett. 89, 121,113 (2006).

[6] A. Figotin, and I. Vitebsky. Nonreciprocal magnetic photonic crystals. Phys. Rev. E63, 066609 (2001).

[7] A. Figotin, and I. Vitebskiy. Electromagnetic unidirectionality in magnetic photonic crystals. Phys. Rev. B67, 165210 (2003).

[8] S. Khartsev and A. Grishin. High performance magneto-optical photonic crystals. J. Appl. Phys. 101, 053,906 (2007).

[9] S. Kahl and A. Grishin. Enhanced Faraday rotation in all-garnet magneto-optical photonic crystal. Appl. Phys. Lett. 84, 1438 (2004).

[10] L. D. Landau, E. M. Lifshitz, L. P. Pitaevskii. Electrodynamics of continuous media. (Pergamon, N.Y. 1984).

[11] A. G. Gurevich and G. A. Melkov. Magnetization Oscillations and Waves. (CRC Press, N.Y. 1996).

[12] A. Figotin and I. Vitebskiy. Gigantic transmission band-edge resonance in periodic stacks of anisotropic layers. Phys. Rev. E72, 036619, (2005).

[13] A. Figotin and I. Vitebskiy. Frozen light in photonic crystals with degenerate band edge. Phys. Rev. E74, 066613 (2006).

[14] A. Figotin and I. Vitebskiy. Slow-wave resonance in periodic stacks of anisotropic layers. Phys. Rev. A76, 053839 (2007). 


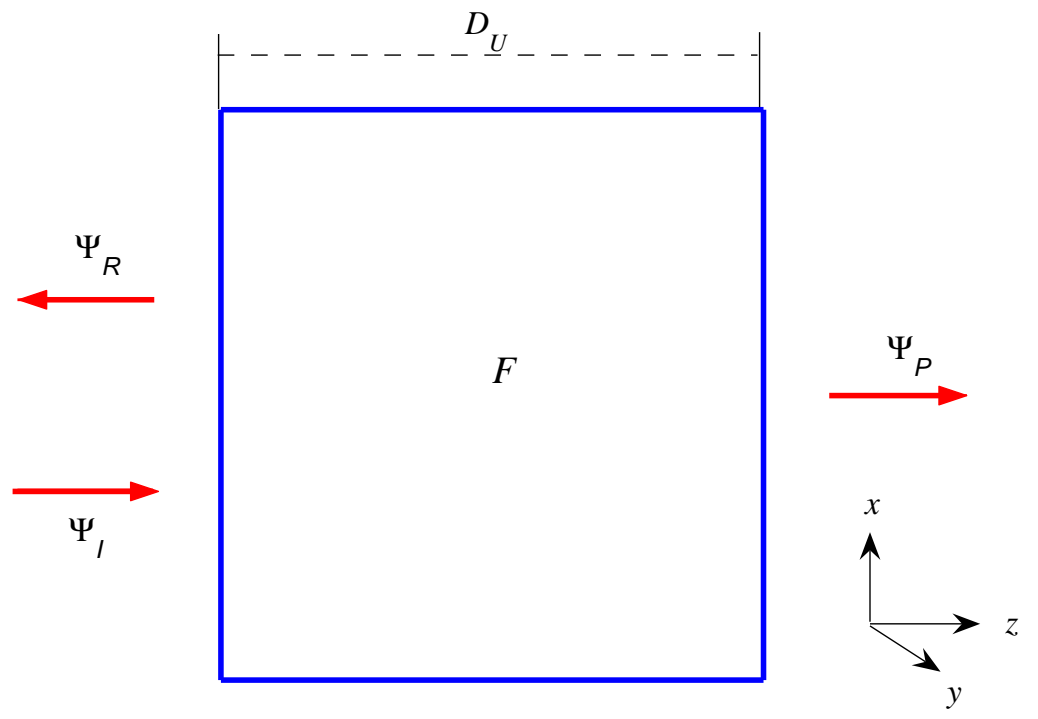

Figure 1. (Color online) Electromagnetic wave propagation through a uniform lossy magnetic slab. The direction of magnetization is normal to the slab. The incident wave $\Psi_{I}$ is linearly polarized with $E \| x$. Due to the nonreciprocal circular birefringence/dichroism of the magnetic material, the reflected wave $\Psi_{R}$ and the transmitted wave $\Psi_{P}$ are both elliptically polarized. 


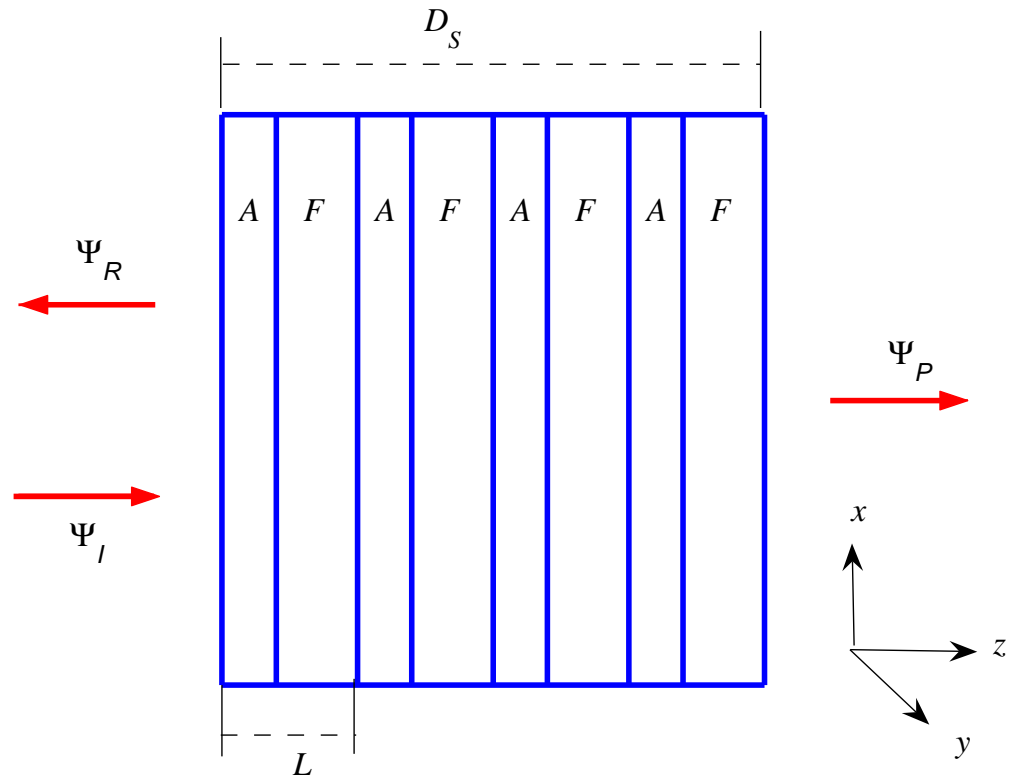

Figure 2. (Color online) The same as in Fig 1 but instead of the uniform magnetic slab we have a periodic layered structure composed of alternate magnetic $(F)$ and dielectric $(A)$ layers. The $F$ layers are made of the same lossy magnetic material as the uniform slab in Fig. 1 $L$ is the unit cell length. 


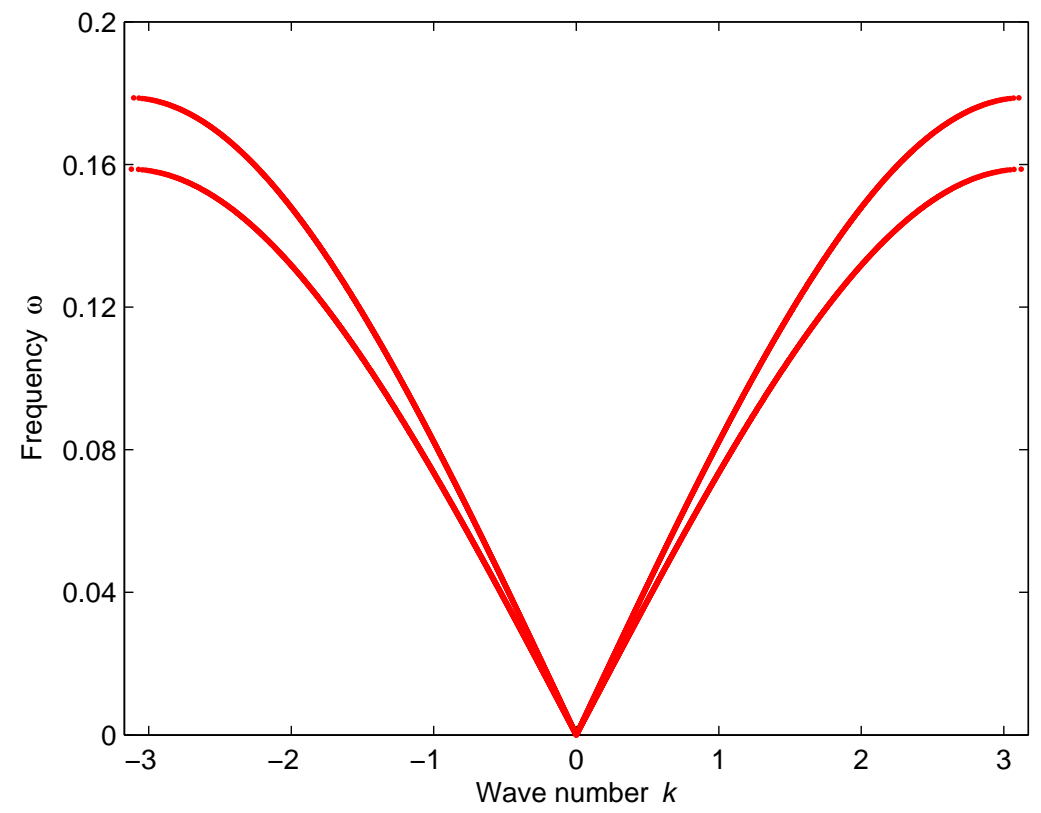

Figure 3. (Color online) The lowest band of the Bloch $k-\omega$ diagram of the periodic layered structure in Fig. 2 with physical parameters specified in (10). The split of the spectral branches is due to the circular birefringence in the magnetic layers. 

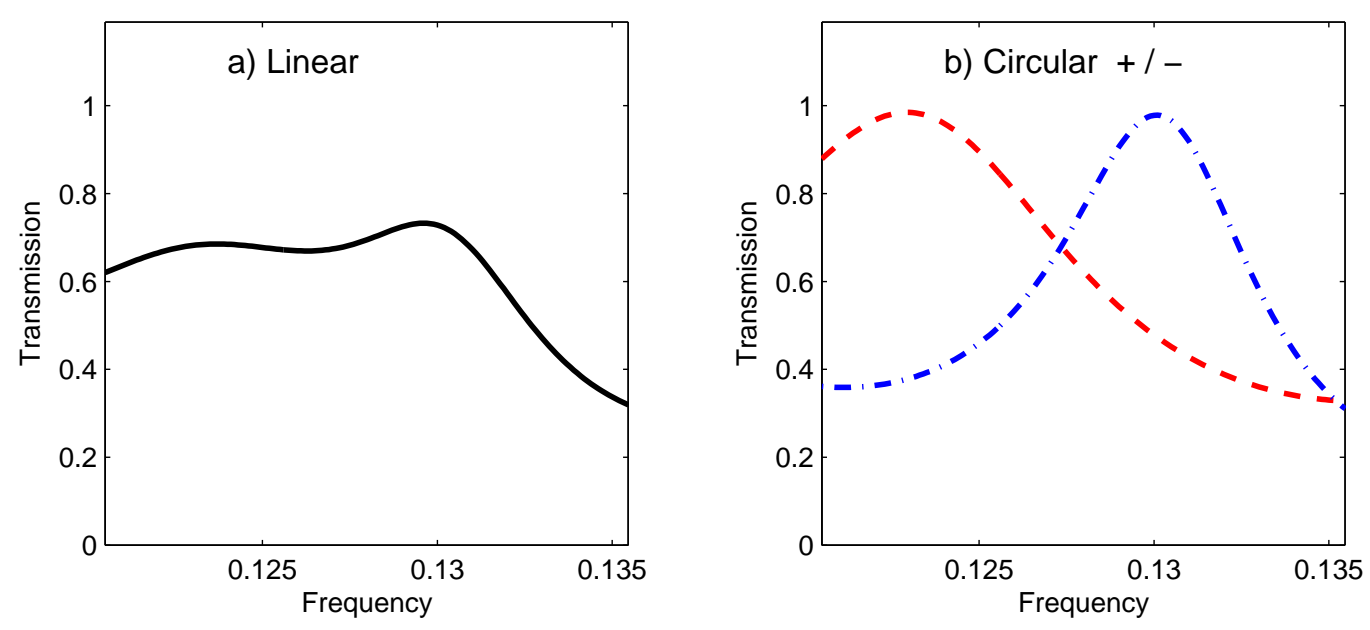

Figure 4. (Color online) Transmission $t$ versus frequency $\omega$ of the periodic magnetic stack in Fig. 22 with the physical characteristics specified in (10): (a) the incident wave polarization is linear. (b) the incident wave polarization is circular. Two transmission maxima on the right correspond to two Fabry-Perot resonances for each of the two senses of circular polarization. At the intersection frequencies of the two curves, the transmitted wave polarization is linear, while at all other frequencies, it is elliptical. 

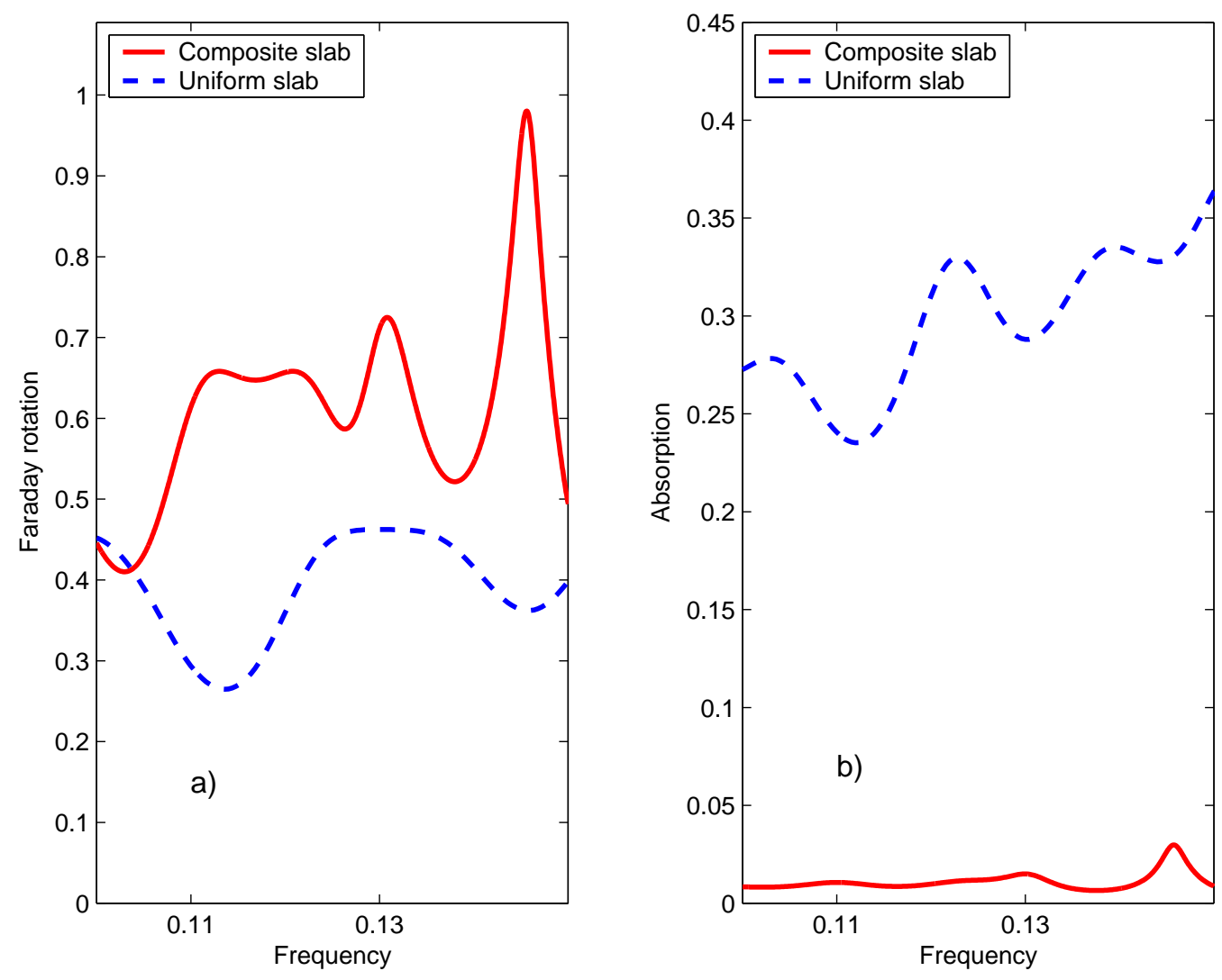

Figure 5. (Color online) Comparative transmission characteristics of the periodic magnetic stack versus the uniform magnetic slab. The physical parameters of both uniform and non-uniform samples are specified in (10): (a) Faraday rotation $|\rho|$ versus frequency $\omega$ of the uniform magnetic slab (the dashed curve) and the periodic layered structure (the solid curve). (b) The respective absorption $a$ versus frequency $\omega$. The graphs show that the composite structure produces similar Faraday rotation as the uniform magnetic slab, while greatly reducing the absorption in a relatively broad frequency range. The frequency range shown coincides with that in Fig. 4 


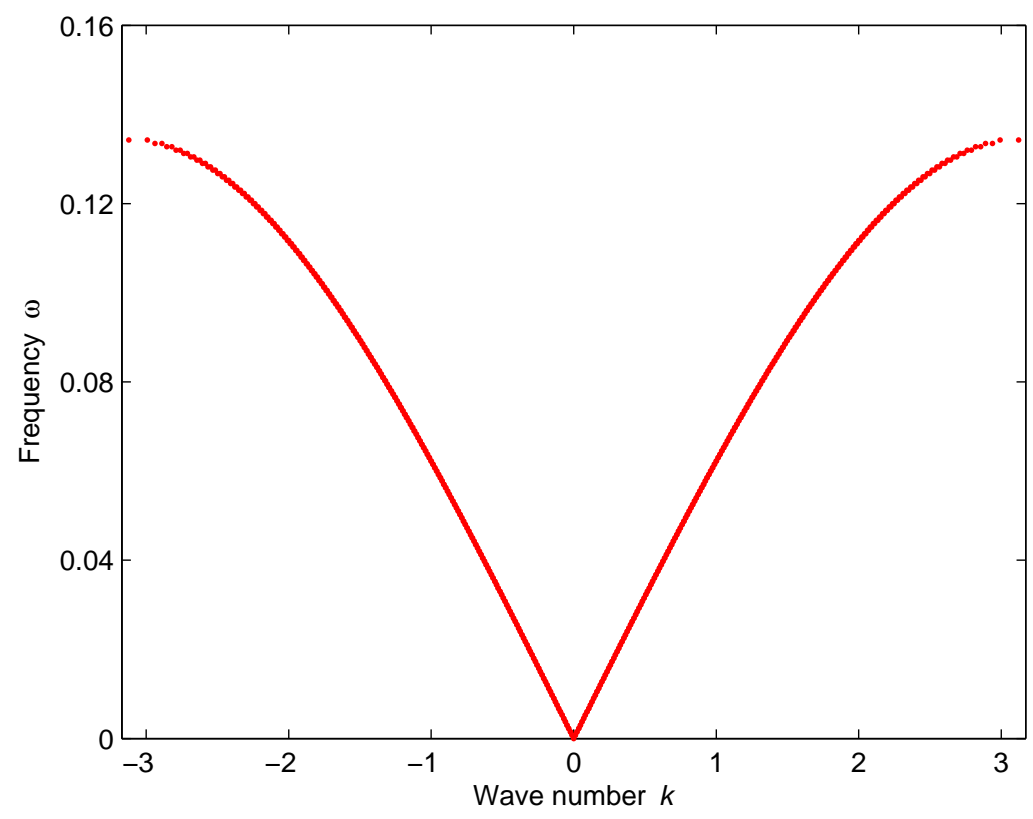

Figure 6. (Color online) The lowest band of the Bloch $k-\omega$ diagram of the periodic layered structure in Fig. 2 with the physical parameters specified in (11). Due to the relatively weak circular birefringence in magnetic layers, the split of the spectral branches is not visible, as opposed to the case (10) shown in Fig. 3 

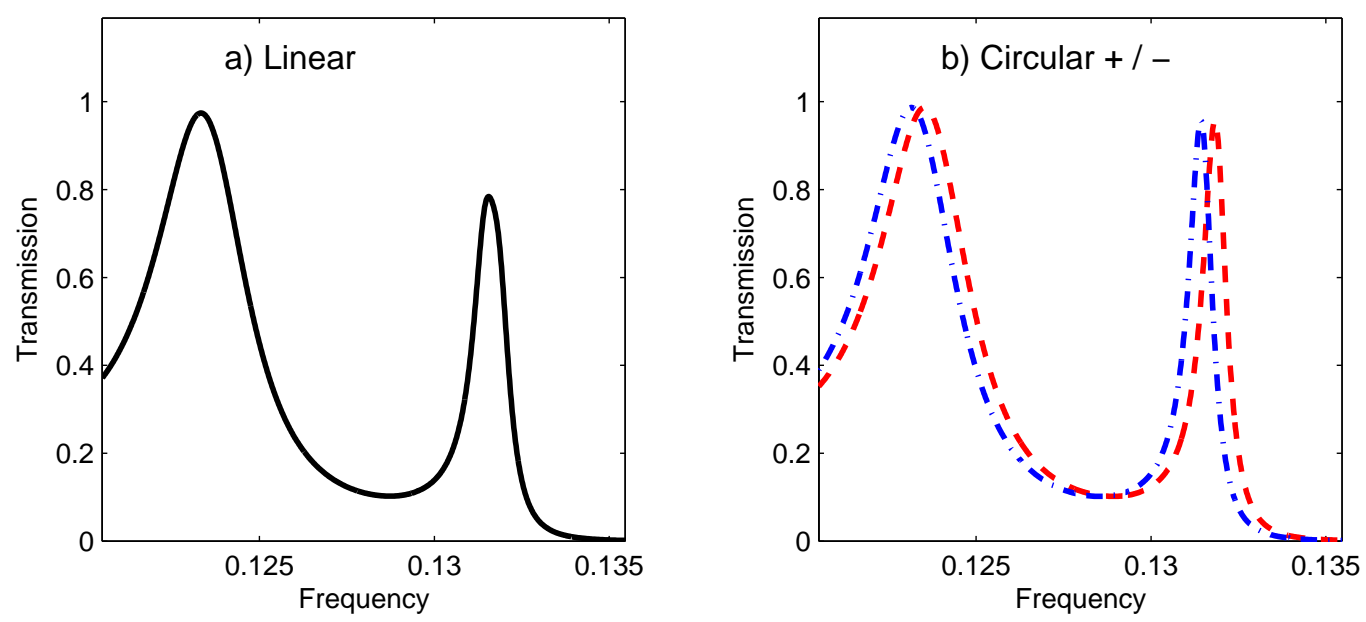

Figure 7. (Color online) Transmission $t$ versus frequency $\omega$ of the periodic magnetic stack in Fig. 22 with physical parameters specified in (11): a) the incident wave polarization is linear. b) the incident wave polarization is circular. Two transmission maxima on the right correspond to two Fabry-Perot resonances for each of the two senses of circular polarizations. At frequencies corresponding to the curve intersection, the transmitted wave polarization is strictly linear (a pure Faraday rotation). At all other frequencies, there is a small ellipticity. 

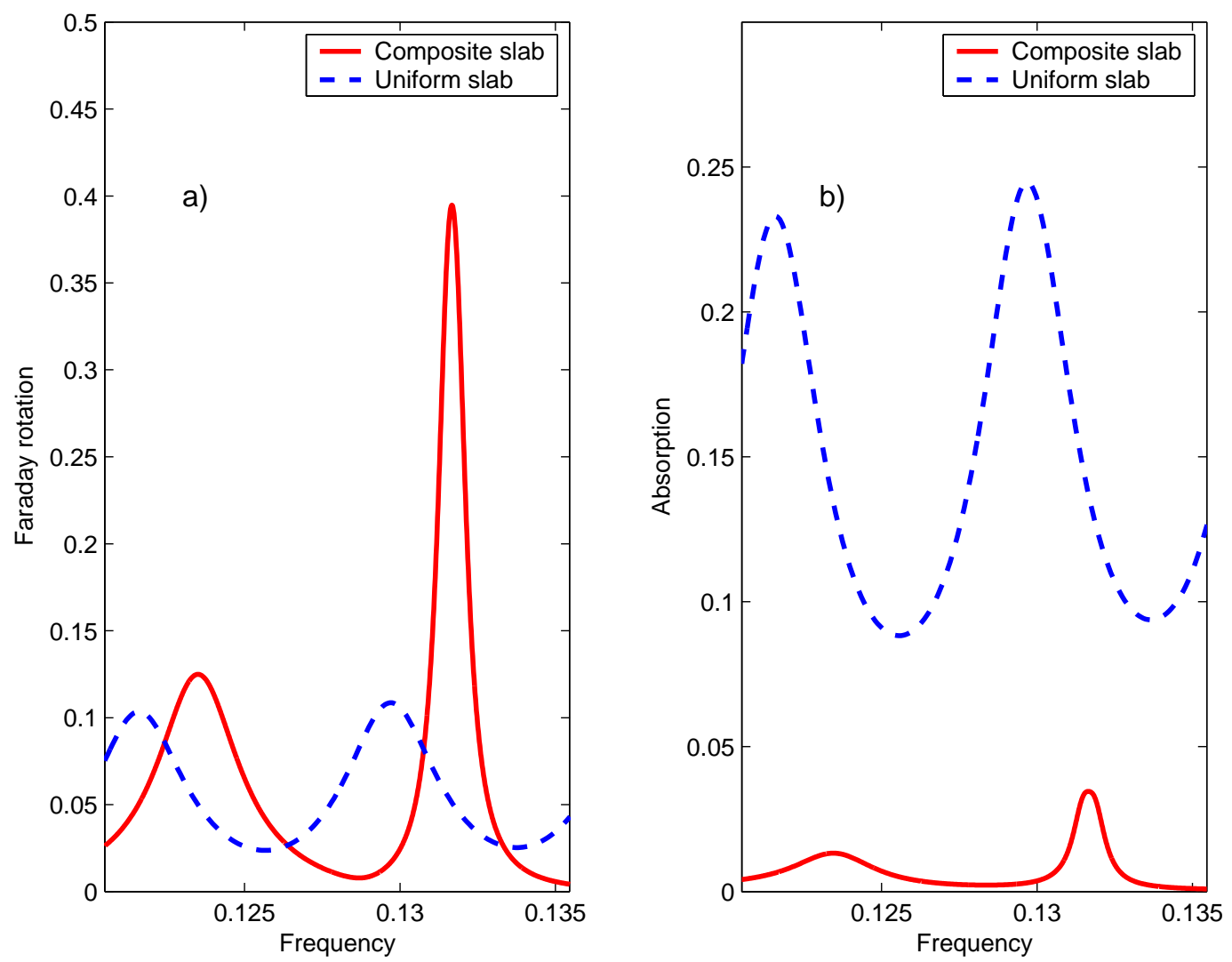

Figure 8. (Color online) Comparative transmission characteristics of the periodic magnetic stack versus the uniform magnetic slab. The physical parameters of both uniform and non-uniform samples are specified in (11): (a) The Faraday rotation $|\rho|$ versus frequency $\omega$ of the uniform magnetic slab (the dashed curve) and the periodic layered structure (the solid curve). (b) The respective absorption $a$ versus frequency. The graphs show that at the frequency of transmission band edge resonance, the composite structure produces much stronger Faraday rotation than that of the uniform slab, while greatly reducing the absorption. In addition, the periodic stack thickness is 5 times smaller than that of the uniform magnetic slab. 